\title{
Historical Changes, Status and Value Utilization of Three Guanhan Roads
}

\author{
Junxia Jia \\ School of Historical Culture and Tourism \\ Xi'an University \\ Xi'an, China 710065
}

\begin{abstract}
Baoxie Road, Chencang Road and Tangluo Road were important paths for economic and cultural exchanges between the ancient Central Plains and southwest China, which were called "Three Guanhan Roads" by the Qin Merchants in the Ming Dynasty. In history, although Three Guanhan Roads have been changed several times and once been silent, their important historical position, rich cultural value and unique ecological tourism resources are of great significance to the development of Guan-Han historical culture and tourism culture industry.
\end{abstract}

Keywords-Three Guanhan Roads; historical changes; status; ecological resources; value utilization

\section{INTRODUCTION}

Hanzhong region is rich in products, once known as "the kingdom of heaven" and famous as "the birthplace of the Han Dynasty" in the world. Since ancient times, Hanzhong has been a place of strategic importance for the Central Plains Dynasties to manage the southwest China. Overlooking the map of China's history, the Qinling Mountains stretch across the central, not only causing a huge difference in the climate between North and South China, but also becoming a natural barrier to North-South traffic. At this background, the wise ancestors have developed a series of ancient roads in the valleys of the Qinling Mountains. These roads, like blood veins, closely linked the Guanzhong Basin and the southwest region. Among these ancient roads, Chencang Road, Baoxie Road and Tangluo Road, known as "Three Guanhan Roads"1, have important military, economic and cultural status in history. They are the crystallization of the wisdom of the Chinese ancestors and the precious wealth left to us. In today's construction of economic power and vigorous development of tourism and cultural industry, it is of great significance to sort out the historical changes of "Three Guanhan Roads" and explore its military, economic and cultural status for the full development and utilization of its value and the leap-forward development of tourism and cultural industry in the Guan-Han region.

There are two views about "Three Guanhan Roads": one is that they refer to Chencang Road, Baoxie Road and Tangluo Road; the other is that they refer to Chencang Road, Baoxie Road and Ziwu Road. This article takes the former view.

\section{THE HistoricAl CHANGES AND MILITARY AND ECONOMIC STATUS OF BAOXIE ROAD}

Baoxie Road was the earliest link between Guanzhong and Hanzhong. It was also known as Xiegu Road. It starts from Baogu Pass (near the Dazhong Temple in Hanzhong) in the south, and reaches to Xiegu Pass (Meixian Xieyu Pass), along the two rivers of Baoxie and through two valleys of Baoxie, hence the name. As early as in ancient times, the Central Plains Chinese people exchanged with the southwestern nationalities through Baoxie Road. Hanzhong was the forefront of the exchange and integration of the Chinese nation and the southwestern nationalities.

During the period of Yao, Shun and Yu, Mr. Bao assisted $\mathrm{Da} \mathrm{Yu}$ to control the water. After more than ten years, he spared no pains and bore hardship without complaint. Because of his achievements, he was conferred to be king of Bao Area, located in the north of today's Hanzhong Hanjiang. Today, there are still relics of the Bao State relics in the Hedongdian Town, Hanzhong Hantai District and Mianxian Baocheng Town. There are records of the communication and combination of Family Zhou and Family Bao and Southwest Nationalities in Shang Shu. At the end of the Shang Dynasty, King Wu of Zhou led the troops to attack King Zhou of Shang Dynasty. The tribal leaders of Yong, Shu, Qiang, Mao, Wei, $\mathrm{Lu}$, Peng and Pu led their soldiers and followed King Wu to attack King Zhou ${ }^{[1]}$ P234-235. Yong was located in today's Hubei Fangxian; Shu in the west of today's Sichuan; Qiang in the southeast Gansu; Mao in the border areas of Sichuan and Gansu; Lu in Hunan Nanzhang; Peng in the east of Gansu Zhenyuan; and $\mathrm{Pu}$ between Hubei Yunxian and Henan Dengxian. It can be seen that people of Zhou Dynasty maintained a good and close relationship with the southwest ethnic groups. If there was no Baoxie Road, it was impossible for people of Zhou Dynasty to communicate with the southwest ethnic groups.

At the end of the Western Zhou Dynasty, Bao Xiang, the emperor of the Bao State, was appointed as senior official of King You of Zhou Dynasty. He was imprisoned because he advised frankly and offended the king. His son Hongde gave the king a beauty called $\mathrm{Bao} \mathrm{Si}$ and redeemed his father, which caused the story of "joking the feudal princes with war flames". After Quanrong occupied Haojing, the Bao State was destroyed, as well as Family Bao. A branch of Family Zheng came to Hanzhong from Baoxie Road and established 
Nanzheng. According to the record of Shuijingzhu - Mian Shui, quoted from Qijiu Biography, "the name of Nanzheng started from Duke Huan of Zheng State, Duke Huan was killed by Quanrong and his people escaped to the south, so people called it Nanzheng [2] (P2311). Nanzheng before Qin Dynasty was ancient Bao State, occupying most of today's Hanzhong region. The ruling government was between today's Hantai and Mianxian.

During the Warring States period, Hanzhong, the northern part of the Hanjiang River belonged to the Qin State, and the southern part of the Hanjiang River belonged to Ba and Shu respectively. Sima Cuo, the general of Qin Dynasty, put forward to attack $\mathrm{Ba}$ and Shu. He thought $\mathrm{Ba}$ and Shu were fertile and rich, and "to get the land and wealth could expand the territory of their country and enrich people and raise army" [3] (P117). Moreover, Ba-Shu land was in the upper reaches of Jing and Chu. "To get Shu helps get Chu, and unify the whole country if Chu was destroyed" [4]. In the fifth year of King Zhou Shenliang's reign (316 BC), the Qin State sent Zhang Yi and Sima Cuo to attack Ba (east of today's Sichuan) and Shu (west of today's Sichuan) through Baoxie Road. Sima Cuo defeated Shu and connected Baoxie Road, from Guanzhong to Hanzhong, with Jinniu Road, entering into Sichuan from Hanzhong. The passage from Guanzhong to Hanzhong to Sichuan was formally linked together. The connection of Baoxie Road and Jinniu Road strengthened Qin's military control over Ba-Shu area, and the economic and cultural exchanges between Qin and Shu became closer.

The name of Hanzhong began in the period of King Huiwen of Qin Dynasty. Qin merged it with Nanzheng of Qin Dynasty and established Hanzhong Prefecture after getting Hangzhong of Chu Dynasty [5] (Vol. 5 Qin Ben Ji P207). The ruling government of Hanzhong County was in Xiecheng (today's Shaanxi Ankang), including Hanzhong. Today, Hanzhong is still called Nanzheng. Hu Sanxing in Zizhi Tongjian held that Hanzhong of Chu Dynasty was not today's Hanzhong. It referred to the six states of Jin, Yang, Jun and Fang and other places. He quoted from Shu Jian: "Nanzheng is Nanzheng and Hanzhong is Hanzhong. Nanzheng refers to ancient Bao State. Before Qin getting Shu, Qin first get Nanzheng. Hanzhong refers to the six states of Jin, Yang, Jun and Fang, lasting six hundred li. After Qin got Hanzhong, Qin set Nanzheng as county, unifying Nanzheng and Hanzhong, which was the beginning" [6] (Vol. 9, P305). On this basis, we can infer that Qin was attacked from two ways, Wuguan and Nanzheng, to capture the middle reaches of the Hanjing River. Before the Han Dynasty, Baoxie Road was the most important passageway to enter Hanzhong from Guanzhong. It was the strategic thrust of Qin's control of Ba-Shu in the South and Jing-Chu in the east. Its military strategic position was selfevident.

In the period of Two Han Dynasties, the central government had carried out three large-scale renovation of Baoxie Road. The first time was during the Yuanshou Years of Emperor Wudi of Han Dynasty (122 BC - 117 BC). Hanzhong Prefecture Chief Zheng Qiong organized peasants to build more than five hundred li of trestle road. The second time was during the Yongping Years of Emperor Ming in the Eastern Han Dynasty. The Eastern Han government further improved the facilities, such as plank road, bridge and post station. According to the rubbing book of Cliff Inscription about Building Baoxie Road of Chu Jun, Hanzhong Prefecture Chief Chu Jun called up two thousand six hundred and ninety people in Guanghan, Shujun and Bashan through an imperial edit to build Baoxie Road"2, and excavated the Shimen Tunnel creatively. In the decades later, the road was destroyed and people transferred to Ziwu Road. The third time was in the fourth year of Emperor Yanguang of the Eastern Han Dynasty (125). The government of the Eastern Han Dynasty renovated the Baoxie Road, and the imperial court "commanded Yizhou Cishi to stop Ziwu Road and open up Baoxie Road". It quoted from San Qin Ji: "Baoxie was the names of the valleys of Hanzhong. The south valley called Bao and the north valley called Xie. It lasted about seven hundred li" [7] (P251). In the Two Han Dynasties, Baoxie Road was of great significance for the Central Plains Empire to control and manage the southwest. From the military point of view, the renovation of Baoxie Road has strengthened the control of Han Dynasty over Ba-Shu area and was also conducive to the Han Dynasty's military conquest of Yelang. From the economic and cultural point of view, the restoration of Baoxie Road has really linked Chang'an with the Southwest Silk Road, and strengthened the economic and cultural ties among the Central Plains, Southwest China, Southeast Asia and India. Shi Ji - Huozhilie Biography recorded "the roads last thousands of li and can connect to everywhere. But single Baoxie Road can yoke the pass" [5] (Vol. 129, P3262). Important resources, such as grain, silk, salt and copper, in Hanzhong and Sichuan were continuously transported to Guanzhong and scattered throughout the country through this road. The culture of the Central Plains also flowed into Ba-Shu area through this way, and Baoxie Road became the main channel of economic, trade and cultural exchanges between the Central Plains and southwest China.

During the first years of the Northern Wei Dynasty (504508), Yang Zhi, the governor of two states, Liang and Nanqin, submitted to the imperial court to repair Baoxie Road, which was actually in charge of by Jia Sande. This renovation changed the previous route of Baoxie Road. The north entrance was changed from Xie Valley into Yuezibai Mountain, Chaiguan Ridge to Huiche and passed Fengxian and Sanguan and to Chencang. In fact, the south section of Baoxie Road was merged with the north section of Chencang Road. The new route has laid a foundation for Lianyun Road. Shimen Inscription ${ }^{3}$, caved at the Shimen Cliff of Baoxie Valley, is the true portrait and great praise of the grand roadopening event.

In the Tang and Song Dynasties, people basically used the above route of Baoxie Road and further renovated it. In the fourth Kaicheng Year of Emperor Wenzong of Tang Dynasty (839), Guirong, Jiedushi of West Shannan Road, repaired ancient Qin-Shu road, north to Sanguan, South to Jianmen,

Cliff Inscription about Building Baoxie Road of Chu Jun, commonly known as Da Kai Tong or Kai Dao Stele, is one of the Thirteen Shimen Articles. There are 16 lines, 5 to 11 words on each line. The maximum notes now we see are only 124 words.

Shimen Inscription, full name Taishan Yangzhi Recovering Shimen Inscription, is now hidden in the Hanzhoung Museum. 
lasting thousands of li. It basically connected Guanzhong and Hanzhong. Liu Yuxi praised his achievement in Event of Repairing West Shannan Road: "travelers can forget their tiredness; spanking runner can slow down; passengers carrying family members can settle down; passengers with cargoes can be easy without burdening their shoulders; walkers can ease their feet and riders cannot wear their horseshoes. They all talked about the benefit of the road publically and privately, and people loved to hear it" [8] (P79807981). In the second Guangqi Year of Emperor Xizong of Tang Dynasty (886), Li Keyong conquered Chang'an and Emperor Xizong fled to the south. Because Xingyuan (Hanzhong) Jiedushi burned down the Pavilion Road, he repaired ancient Baoxie Road to Hanzhong. According to Zizhi Tongjian, "he commanded Jin Hui and Shen Ce to lead armies and stationed on Heishui ${ }^{4}$ to repair the road for passing" [6] (Vol. 256 P8333). In the early Song Dynasty, although Dayi Road connected Guanzhong and Shudi, Xiegu Road in Tang Dynasty was not abandoned. After the tenth Xining Year of Emperor Shenzong of Song Dynasty (1077), Xiegu Road (Lianyun Road) became one of the economic and cultural arteries connecting the Central Plains and southwest China.

During the Hongwu Years of Ming Dynasty, Lianyun Road was formally completed. This route was winding like a cloud ladder, passing Qipan Ridge and Jitou Pass, hence its name. It connected Baoxie Road in the south, and turned to the northwest, passed Bali Pass (ancient Xiaowan Station), Qinglong Temple (ancient Heilong Station), Yuexinkai Ridge (ancient Dengpo Station), Qingyang Pu (ancient Qingshui Station), Huamei Pass, Datan, Anshan Post (Anshan Bay), and entered Fengxian County and Chencang Road. Since then, the road has been basically finalized, which was repaired twice by the imperial court during the Wanli period of the Ming Dynasty and the Kangxi period of the Qing Dynasty. In the third year of Kangxi's reign (1664), according to Dang Chongya's Great Minister Mr. Jia's Road Repairing Story, Shaanxi governor Jia Hanfu and Hannan Patrol Envoy jointly planned to mobilize the Fengxiang Fund Raising and Labor Collection to carry out the last large-scale renovation of Xiegu Road in the Tang Dynasty. The project totaled 200 li. The Library of Congress of the United States has an anonymous collection of Shaanxi Shudao Map of Qing Dynasty. Mr. Feng Suiping has made a textual research on it based on historical records and existing steles. He believed that this map was first drawn between the fiftieth Qianlong year (1765) and the fortieth Qianlong year (1775). The area covers Baoji County (today's Baoji City), Fengxian, Liubating, Baocheng, Mianxian, Ningqiang State the Qipan Pass's Lianyun Road adjacent to Qin-Shu. Lianyun Road is an important way to link the Central Plains and southwest China in the Qing Dynasty. The north station, also called Qin Station, includes area from Baoji County to Baocheng County. Baoji Yimen was the entrance of the station, and Baocheng is the exit of the station; to the south of Mianxian is the south station, also

Fangyu Shenglan · Lizhou East Road · Xingyuan Fu: "Heishui River started from Chenggu County, the northwest of Taibai Mountain, and flew into Hanzhong in the south. Zhuge Liang wrote: 'starts from Nanzheng in the morning and arrived and stayed at Heishui at dusk.'" Lizhou West Road . Jiao Zhou: "Heishui starts from the central of Qiang." called Shu station. He also pointed out that Shaanxi Shudao Map was to reflect Lianyun Road in Shaanxi Province in the

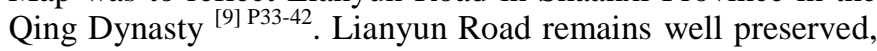
and Lianyun Trestle Road is now one of the eight scenic spots in Fengxian, still silently telling of the past prosperity.

With the aid of "Kaizhong System" of the Ming government, the Shanxi Merchants Gang grew rapidly and almost controlled the economic exchanges between the southwest and the Central Plains. There were Shaanxi guildhalls everywhere in the southwest Dayi. People in Sichuan once said, "tofu, old Shaan and dogs are all over the world." As an important passageway between Sichuan and Shaanxi, Baoxie Road has prominent economic functions. The merchants of Guan and Shaanxi processed raw tea of Hunan and Jiangxi into brick tea and sold it to the southwest. They transported silk, medicinal materials, salt, ore and fur from the southwest to the whole country. They also introduced social customs, wine-making techniques and dietary culture of the Central Plains into the southwest through this way, which greatly changed the economic and cultural life of the southwest.

\section{HistoricAl CHANGES AND STRATEGIC AND ECONOMIC STATUS OF CHENCANG ROAD}

Chencang Road, also known as Gu Road and Jialing Road, is famous for a literary quotation "openly repair the trestle road in order to pass Chencang Road without being found". It started from Baoji and entered into Sanguan to the southwest, and along the upper reaches of the Jialing River it reached to today's Fengxian and then turned to the southwest along the valley of Jialing River, passed today's Liangdang (ancient road of Han Dynasty, Huixian (Hechi of Han Dynasty) to today's Lueyang (Jialing Road of Han Daynsty) and connected Jushui Road and reached to Hanzhong, or passed Chenping Road in the territory of today's Lueyang and reached to Ningqiang Da'an Station and connected to Jinniu Road entering into Sichuan.

Chencang Road was first built around the Shang and Zhou Dynasties. In the inscription of "Sanshi Plate", the bronze ware unearthed in Fengxiang County of Shaanxi Province in the early Qianlong years of Qing Dynasty, there are words "conferring to Zhou Road". The San State was located in Baoji and Fengxiang, the south of Weihe River. According to Mr. Wang Guowei's textual research, "Zhou Road" refers to Zhougu Road, that is, Chencang Road ${ }^{[10](\mathrm{P} 877)}$. Once it was abandoned because Chencang Road was far away from Ankang, the seat of ruling government of Hanzhong Prefecture and passages needed to make a detour during the Warring States Period.

During the struggle for hegemony between Chu and Han Dynasties, the neglected Chencang Road was rediscovered, which played a vital role in the establishment of the Han Dynasty. In the first month of the first year of the Han Dynasty $^{5}$, Xiang Yu set Liu Bang as the king of Han, governing Ba, Shu, and Hanzhong, taking Nanzheng (today's Hanzhong) as capital. Liu Bang took Zhang Liang's advice

In the early Han Dynasty, the summer calendar was adopted, and October was the beginning of the year. 
and burned down Baoxie Road to show Xiang Yu that he was no longer coveting Guanzhong, so as to win the trust of Xiang $\mathrm{Yu}$. King of Han Liu Bang, after a period of recuperation in Nanzheng, used Han Xin as general to fight for Guanzhong again. Han Xin used the diversion tactic and openly built Baoxie Road to confuse the enemy but secretly used Gu Road (today's Fengxian), and occupied Chencang and Haozhi (today's Qianxian), and then Feiqiu (today's Xingping), and arrived Xianyang to the east. Thus he occupied Guanzhong and laid the foundation for the Great Han Dynasty. Xin Qiji, a poet in Song Dynasty, praised Hanzhoung for saying "Hanzhoung opens Han Dynasty".

During the period from the beginning of the Northern Wei Dynasty to Yongping Years, Lianyun Road was built by the Northern Wei Dynasty to link the north and the South horizontally in Shaanxi and Sichuan. Lianyun Road connected Baoxie Road to the south, passed An'shan Station, Zhangliang Temple, Liufeng Pass, Xinhongxia, Nanqi Mountain and Fengxian and reached to Chencang Road. The construction of Lianyun Road enlarged the transportation capacity of Chencang Road and further strengthened the economic and trade exchanges between Guanhan and Sichuan and Shaanxi.

Chencang Road was the longest, but relatively flat way among the Three Guanhan Roads, and a long way overlapped with the waterway of Jialing River. It could borrow the convenience of the waterway of Jialing River, so after the Wei and Jin Dynasties, Chencang Road became the most important traffic between Guan and Han. With stable society during the Sui and Tang Dynasties, traffic was frequent among Chang'an, Nanzheng and Chengdu. The construction of Lianyun Road increased the status of Chencang Road and became an important road between Sichuan and Shaanxi. Along Jialing Road in Qinling Mountains, there were more than 15 stations in less than 100 kilometers from Sanguan to Baoji.

In the two Song Dynasties, Chencang Road was the front line of Song and Jin's confrontation. Wu Jie and Wu Lin led the Song Army to defend Great Sanguan and Xianren Pass against the Jin Army. With the support of the people and the volunteer army, the Song Army won more with fewer victories. They successively won two great victories in Heshangyuan and Xianren Pass which were listed as "the 13 battle achievements of the Southern Song Dynasty"6. After $\mathrm{Wu}$ Jie's death, Wu Lin led the army and fought against the Jin soldiers in Yanjiawan, which was better than the two battles in Heshangyuan and Xianren Pass. Hu Shi, Xuanfu Minister of Sichuan-Shaanxi and Bachelor of Baowenge, thought that "the battle in Yanjiawan had not been so successful since the army was used in Sichuan and Shaanxi" [11] (P2285). With the efforts of Wu brothers and soldiers and

6 In the second Qiandao year of Emperor Xiao of the Southern Song Dynasty (1166), Emperor Xiao commended the remarkable military achievements to conquer the south and found Yan in order to inspire morale. Excepting for commending the four battles of Mingzhou, Dayi Town, Heshangyuan and Xianren Pass in the tenth Shaoxing Year (1140), he also commended the battle of Shunchang and the eight battles of Tangdao, Zaojiaolin, Xupuqiao, Caishi, Caizhou, Cihu, Queshan and Haizhou at the end of Shaoxing, and designating them as 13 battle achievements since Zhongxing. civilians in the Song Dynasty, the Western defense line of the Song Dynasty was eventually consolidated in the line of Great Sanguan, which played a very important role in ensuring the peace of Hanzhong, Sichuan and the territory of Southern Song Dynasty.

During the Ming and Qing Dynasties, extremely rich Qin merchants controlled the ancient tea-horse road to southwest China. The trade and finance in Yunnan, Guizhou and Sichuan were all in the hands of Shaanxi merchants. Chencang Road played a very important role here. Up to now, there are still remains of Shaanxi Guildhalls in Chengdu, Ya'an, Kangding and Changdu. Shaanxi Street (Shaanxi Road) is mostly a local commercial center. Qin merchants purchased tea from Sichuan and Hunan and transported them to Jingyang to process into brick tea and shipped them back to Sichuan and sold them to Tibetan areas. Musk, cordyceps, fritillaria, gold, wool and fur from Yunnan, Guizhou and Sichuan were transported to Guanzhong and distributed throughout the country. Chencang Road also thrived because of the tea-horse trade, which was densely populated and economically active. In the Qinling Mountains in Baoji, Fengxian, Huixian County and Lueyang, there were a lot of ancient towns and there are still more than 20 ancient towns of the Ming and Qing Dynasties, scattered in the mountains. The south section of Sichuan-Shaanxi Highway from Hanzhong to Baoji built in the period of the Republic of China overlaps with Baoxie Road, and most overlaps with Chencang Road. The route of Baoji-Chengdu Railway, built in the early days of the founding of the People's Republic of China, almost took the route of the ancient plank road for thousands of years. This proves that the Qinling Trestle Roads is the crystallization of the Chinese nation's experience and wisdom. It is worth exploring and carrying forward the advanced national culture.

\section{THE HistoricAl CHANGES AND THE MILITARY AND ECONOMIC STATUS OF TANGLUO ROAD}

Tangluo Road was named due to its south mouth located in the mouth of Yangxian Tang River in Hanzhoung, and the north entrance located in the West Luo Valley in Zhouzhi County. From the map, Tangluo Road was almost a straight line between Xi'an and Hanzhong. But because it was the closest to Taibai Mountain, the main peak of the Qinling Mountains, and travelers had to cross five or six high mountains above 3000 meters above sea level. So, Tangluo Road was the fastest and most dangerous ancient road among Guanhan roads. Poet Sage Du Fu ever wrote "twenty-first families went to Shu together, but only one family left Luo Valley. When she said that, her daughters were gnawing their arms. They turned to Qinyun and cried" [12] in Three Jue $\mathrm{Ju} \cdot$ Yuzhou Killing Cishi the Year before Last. It can be seen the dangerously steepness of this road.

The construction of Tangluo Road has no textual research. It was first used by the ancestors to collect medicine and hunt. It was the fastest and most concealed road between Guan and Han States, it began to be used for military purposes around the end of the Eastern Han Dynasty. During the Three Kingdoms Period, Liu Bei was conferred as the king of Hanzhong. He took Hanzhong as base to attack the Cao and Wei States in the north. Tangluo Road pointed directly to the 
hinterland of Guanzhong and had no obvious reputation, so it was easy to launch surprise attacks.

Folk legends say the construction of Tangluo Road owed to Liu Xiu, which can better prove the fact of Liu Bei's construction of Tangluo Road. In folklore, Emperor Gaozu of Han Dynasty Liu Bang, Emperor Guangwu Liu Xiu, and Emperor Zhaolie of Shu-Han Liu Bei were often confused as the King of Han. It perhaps is because all of them are emperors for the founding of Liu's State. Emperor Gaozu first occupied the west Gansu, and went to the west along Chencang Road and occupied Guanzhong. Emperor Guangwu rose to power Hebei. He sent Geng Yi and Feng Yi to take Guanzhong. Only Liu Bei was based in Hanzhong and faced the powerful $\mathrm{Cao} \mathrm{Cao}$, and defeated him by a surprise action. Zhiguo Stone, Mang Ridge and Hanwang Mountain illustrated the hardships to built Tangluo Road.

In the later period of the Three Kingdoms, Jiang Wei, a general of the Shu state, attacked the north through Tangluo Road. He once arrived the south of Zhouzhi County (former county). General of Wei State Cao Shuang also attacked Shu State through Tangluo Road. General of Wei State Zhong Hui also attacked Shu through this road. After Zhuge Liang's death, the Shu State was in chaos and Tangluo Road was abandoned, which led to the fall of Hanzhong and the eventual destruction of the Shu State. It can be seen that in the Three Kingdoms period, Tangluo Road was only an intermittent military road.

After the establishment of the Tang Dynasty, the national strength was strong and the society was stable. The government of the Tang Dynasty made full repairs to the transportation facilities, including pavement, pass and post of Tangluo Road. At one time, the status of the Tangluo Road was comparable to that of Chencang Road and Baoxie Road. In the mid-Tang Dynasty, "there was one station in every $30 \mathrm{li}$ and one post in every 10 li" in Tangluo Road. The allocation of army, promotion, relegation and debriefing of officials, many business travelers were all through this way. Tangluo Road, passing through old town of Zhouzhi, Foping and Huayang Town, had also experienced a great deal of business and tourism, gathering with goods and flourishing for a time.

After the Tang Dynasty, the political center moved eastward, and the economic status of Guanzhong area was not the same as before, so the Tangluo Road gradually declined. In the Southern Song Dynasty, Guanzhong area was occupied by the Jin State. In order to defend the invade of the Jin State from the south, the regime of the Southern Song Dynasty destroyed part of the transportation facilities of Tangluo Road, until the Ming Dynasty, the Tangluo Road was almost abandoned.

During the Ming and Qing Dynasties, the trade between Sichuan and Shaanxi was frequent. Travelling merchants were dense, and Chencang Road, Baoxie Road and Ziwu Road could not meet the needs of merchant travelers for trade exchange, so Tangluo Road was flourished again. Compared with Chencang Road, the economic value of Tangluo Road is much less than that of Chencang Road, which were mainly small merchant teams, composed of small merchants, but it is still very valuable for the preservation of Tangluo Road.

\section{The Value Utilization of Three GuanhaN ROADS}

Nowadays, the Qinling Trestle Road between Guan and Han has been replaced by modern highways, railways, expressways and airports, but this does not mean that the ancient trestle road has lost its value of existence. With the development of Guan-Han tourism culture industry, the ancient plank road is revitalized.

\section{A. "Three Guanhan Roads" Should Make Use of the Opportunity of Application for the List of World Heritage of Ancient Shu Roads to Build a Path of Culture Along the Way}

There are a large number of historical relics in the "Three Guanhan Roads". The important ones are the ancient passes, ancient towns, the ruins of ancient roads and numerous stone inscriptions on the cliffs. For example, Great Sanguan Ruins on Chencang Road, Mingyue Valley, Wuwang Town Relics (Xianren Pass), "Ode to Fu Pavilion" Stone Inscription, Lianyun Road, "Thirteen Shimen Articles" on Baoxie Road and ancient Huayang town on Tangluo Road. Two places of Guan and Han should work together to restore the reputation of the dusty ancient road.

Ode to Hansan is a representative of the famous cultural relics on Three Guanhan Roads. Ode to Shimen, full name Sili Xiaowei Mr. Yang's Ode to Qianwei, is the fifth article in the thirteen Shimen Articles. It belongs to the first batch of national key cultural relic protection units. Its font is stretching, open, natural, and start stroke, close stroke and movement are characteristic; strokes are thick, writing follows stone trend, moving in a direction freely. It is known as "Cursive in Clerical Script", "the representative of novel and free Han clerical script". Ode to Fu Pavilion, full name Wudu Taishou Li Xixi's Ode to Fu Pavilion on Xili Bridge, belongs to standard Bafeng Han clerical script. It is said it was wrote by Cai Yong, a calligrapher of the Eastern Han Dynasty. It is an important material data of stone inscription on cliff about ancient words, calligraphy and Bafeng Han clerical script of Eastern Han Dynasty. The East Asian countries, especially Japanese and Korean calligraphers, have conducted a multidimensional study of it. Ode to Xixia was from the cliff stele of West Hui'an Biao, located in Gansu Chengxian on the Chencang Road, which were written by calligrapher and painter Qiu Jing. It has very high calligraphy value.

Shu roads apply for the world's natural and cultural heritage, and "Three Guanhan Roads" should take advantage of the opportunity to build a cultural road, actively carry out a more detailed survey of the cultural relics on the roads, and reasonably restore the cultural relics that are conditionally restored.

\section{B. Doing a Good Job in the Protection and Development of the Ancient Towns Along "Three Guanhan Roads"}

"Three Guanhan Roads" stretch across the Qinling Mountains, and there are countless ancient towns in the Qinling Mountains. The green mountains and clear water and fresh air are precious humanized landscape resources. There are ancient towns, including Qingmuchuan, Liuhou, Huayang and Houzhenzi, along these roads, and they have a long 
history and profound cultural background. Some of them have centuries or even thousands of years old buildings, old blocks, simple folkways, unique folk customs, traditional lifestyles and production appliances, like pearls on the plank road, giving off sparkling and attractive charm. Therefore, it is of great practical significance to do a good job in the protection and development of the ancient towns along "Three Guanhan Roads" for building the brand of Qinling tourism and cultural industry.

\section{Relying on Abundant Biological Resources on Qinling Mountains to Create a Green Ecological Corridor}

Qinling Mountains is the natural geographical dividing line between the north and south in China. "Three Guanhan Roads" pass through from north to south. The north of Qinling Mountains is a warm and semi-humid climate. The south of Qinling Mountains is a subtropical humid climate. With great altitude fall, animal and plant resources are extremely rich. The natural landscapes of the north and south are different. For example, there are more than 2000 kinds of seed plants in Qinling Mountains, including ginkgo, unifoliate, larix chinensis, picea neoveitchii, euptelea pleiospermum and other national key endangered protected plants. There are more than 30 species of rare protected animals in the country. The four national treasures, crested ibis, giant pandas, golden monkeys and antelope, are all distributed here, which are rich in biological gene treasures. In addition, the bird resources along the Three Guanhan Roads are also very distinctive, especially along Tangluo Road, which is known as a paradise for birdwatchers to watch all kinds of rare birds. It is urgent to enrich the biological resources and create a green ecological corridor for tourism.

\section{Relying on Complex Natural Landscapes on Qinling Mountains to Create High-end Tourism Routes}

Along "Three Guanhan Roads" and their surrounding, there are snowy mountains, alpine meadows, coniferous forests, broad-leaved forests, plains, swamps and landslides and other landforms, which are ideal places for outdoor sports. With the improvement of people's living standards, high-end outdoor sports will become a steady growth point of tourism. "Three Guanhan Roads" has the dual nature of the human landscape heritage and the natural resources treasure house, so it is particularly urgent to create high-end tourism routes. And the employees shall have a fairly high quality. Firstly, they shall possess humanistic and historical accomplishment, and have a considerable reserve of humanistic and historical knowledge of "Three Guanhan Roads", and help tourist learn knowledge during enjoying it. Secondly, they shall possess certain professional knowledge of geography and biology, so as to provide beneficial guidance to tourists in nature and help tourists appreciate the wonders of the nature. Thirdly, they shall undergo professional rescue training in the field, so that tourists can appreciate the beauty of humanity and nature while having personal protection. Fourth, give all-round industrial cultivation by relying on the humanities, natural resources and using modern media propaganda means.

\section{CONCLUSION}

To sum up, "Three Guanhan Roads" were once important passages for the Central Plains Dynasties to contact the southeast and had a very important military strategic position in history. They were the economic and cultural arteries linking the southwest of the motherland in the stable period of history. They gradually declined with the development of our national industrialization in modern times. However, the vicissitudes of history have endowed them with profound humanistic connotations, and the boundless Qinling Mountains have endowed them with unique ecological resources. It is believed that their value will be brought into full play in today's ever-increasing demand for culture, tourism and natural environment.

\section{REFERENCES}

[1] Jennie Oxley,Rrian Fildes,Elfriede Ihsen,Judith Charlton,Ross Day. Pi Xirui, Checked by Sheng Dongling, Chen Kang, Textual Research on Shang Shu in Modern Text · Mu Shi, Zhonghua Book Company, 1989.

[2] Noted by Li Daoyuan, Sorted by Yang Shoujing and Xiong Huizhen: Shui Jing Zhu Shu, Shanghai Ancient Book Publishing House, 1989.

[3] Copied by Liu Xiangji: Qin First Strategy, Vol. 3, Strategies of the Warring States, Shanghai Ancient Book Publishing House, 1985.

[4] Chang Qu, Noted by Liu Lin: Annotation to Huayang Chronicles, Vol. 3 Shu Chronicles, Ba-Shu Publishing House, 1984.

[5] Sima Qian, Shi Ji, Zhonghua Book Company, 1959.

[6] Sima Guang, Noted by Hu Sanxing: Zizhi Tongjian, Zhonghua Book Company, 1956.

[7] Fan Ye et al. Post Han Shu, Vol. 6, Emperor Shun Biography, Zhonghua Book Company, 1965.

[8] Liu Yuxi. Collections of Liu Yuxi, Vol. 8, Shanghai People's Publishing House, 1975.

[9] Feng Suiping. Re-discussion on Shaanxi Shudao Map, Collected by the Library of United States Congress, Wen Bo, Feb. 2010.

[10] Wang Guowei. Collected Works of Guantang, Vol. 18, Textual Criticisms and Explanations on Sanshi Plate, Zhonghua Book Company, 1959.

[11] Li Xinchuan, Annual Records Since the Founding of Yan Dynasty, Vol. 142, The End Article of October, $11^{\text {th }}$ Shaoxing Year, Zhonghua Book Company, 1956.

[12] Edited by Peng Dingqiu et al., Noted by Zhonghua Book Company: Full Collection of Tang Poems, Vol. 229_11, Zhonghua Book Company, 2011. 